\title{
Percepciones sociales sobre el derecho de autonomía de los pueblos indígenas en Chile
}

\author{
Juan Esteban Fernández \\ Universidad Diego Portales, Santiago, Chile. \\ Email: Juan.fernandezm@mail.udp.cl \\ Claudio Fuentes S. \\ Universidad Diego Portales, Santiago, Chile. \\ Email: claudiofuentes.saavedra@gmail.com
}

\begin{abstract}
Resumen: En este trabajo se estudian las actitudes sociales respectoal derecho de autonomía de los pueblos indígenas en Chile. Basado en un estudio de opinión pública del Centro de Estudios Interculturales e Indígenas (CIIR), se concluye que la demanda por autonomía tiende a ser más relevante entre personas que se auto identifican como indígenas,jóvenes, con niveles de escolaridad baja, que viven en zonas urbanas, de izquierda, y de la macrozona centro y norte. A diferencia de otros estudios que estudian la tolerancia o aceptación de la diversidad étnica, en este estudio se explora el perfil de quienes favorecen/rechazan la autonomía indígena, realizando una contribución a la literatura al especificar que no siempre mayores niveles de escolaridad se asocian con mayor tolerancia a la diversidad y que las demandas por autonomía no necesariamente provienen de los territorios que enfrentan mayor conflictividad.
\end{abstract}

Palabras claves: Autonomía indígena, pueblosindígenas,actitudes sociales, mapuche.

\section{Social perceptionsontheright to autonomyforindigenouspeople's in Chile}

\begin{abstract}
This paper addresses the social attitudes regarding the right to autonomy of indigenous peoples in Chile. Based on a public opinion study of the Center of Intercultural and Indigenous Research (CIIR), the conclusion reached is that demand for autonomy tends to be more relevant among people who identify themselves as indigenous, young people, with low educational levels, living in urban areas, left-wing, and from the center and northern areas of the country. Unlike other studies that study tolerance or acceptance of ethnic diversity, this study explores the profile of those who side with/reject indigenous autonomy, suggesting that not always greater levels of education are linked to higher support for indigenous autonomy. Moreover, we assert that the demands for autonomy are not necessarily linked to those inhabitants of conflict areas.
\end{abstract} mapuche

Keywords:Indigenousautonomy, aboriginalpeoples, social attitudes, 


\title{
Percepções sociais sobre os direitos de autonomia dos povos indígenas no Chile
}

\begin{abstract}
Resumo: Neste trabalho, estudamos atitudes sociais em relação ao direito de autonomia dos povos indígenas no Chile. Com base em um estudo da opinião pública do Centro de Estudos Interculturais e Indígenas (CIIR), conclui-se que a demanda por autonomia tende a ser mais relevante entre as pessoas que se identificam como indígenas, jovens com baixos níveis de educação, que vivem em zonas urbanas, da esquerda e da grande zona do centro e do norte do país. Ao contrário de outras pesquisas que estudam tolerância ou aceitação da diversidade étnica, nesta pesquisa explora-se o perfil daqueles que favorecem/rejeitam a autonomia indígena, contribuindo para a literatura ao salientar que nem sempre maiores níveis de educação estão associados a uma maior tolerância à diversidade e que as demandas por autonomia não são necessariamente provenientes dos territórios que enfrentam maior conflitividade.
\end{abstract}

Palavras-chaves: Autonomia indígena, povos autóctones, Chile, atitudes sociais, mapuche.

\section{Introducción}

Uno de los temas más candentes de las últimas décadas se refiere a las luchas políticas y sociales sobre el reconocimiento de pueblos indígenasen diferentes partes del planeta. Esta lucha ha considerado avances desiguales en algunos Estados y un progresivo reconocimiento en el plano internacional. Para el caso de América Latina se observa una transformación significativa — aunque también desigual—en materia de reconocimiento constitucional en las últimas dos décadas. La temática involucra una dimensión cultural sobre la revisión de concepciones tradicionales de los “estados-nación”, una dimensión material respecto de la lucha por recuperar territorios y espacios que les pertenecían a los pueblos indígenas, y una política, en cuanto al reconocimiento de la autodeterminación de dichos pueblos respecto de las decisiones que les son propias.

En este trabajo abordamos los factores que determinan las percepciones sociales en torno a la autonomía de los pueblos indígenas en Chile. Operacionalizamos el concepto de "autonomía" considerando tres dimensiones: la aceptación que los indígenas puedan controlar ciertos territorios, que puedan autogobernarse, y que tengan una representación política en el Congreso Nacional. Así, mientras una importante parte de la literatura se ha centrado en el estudio de las relaciones interculturales a partir del estudio de la aceptación de un "otro" - tal como el debate teórico a continuación dará cuenta -, en este trabajo se examina la aceptación de ceder poder político a los pueblos indígenas. Evaluamos entonces la existencia o no de una aceptación social sustantiva, lo que nos lleva a dialogar con una literatura que ha examinado las dimensiones políticas de la autodeterminación en otros contextos. 
La investigación de carácter cuantitativa concluye que las variables determinantes son la edad, la auto identificación étnica, el lugar de residencia, la posición ideológica, el nivel de escolaridad y la macrozona de residencia. Así, los jóvenes, aquellos que se auto identifican como indígenas, que residen en centros urbanos, que son de izquierda, que habitan en la zona norte y centro de Chile, y con bajos niveles de escolaridad son más propensos a aceptar la autonomía indígena.

El trabajo tiene implicancias teóricas y prácticas. Teóricamente comprueba hipótesis relacionadas con algunas variables que la literatura había considerado como relevantes para abordar esta temática (particularmente la edad, lugar de residencia, y posición política). Asimismo, contribuye a distinguir entre lo que son las percepciones respecto de la convivencia con un otro, de aquellas percepciones que se asocian a ceder poder a otro.Por ejemplo, mientras algunos autores que abordaremos a continuación indican que el nivel de escolaridad es un buen predictor de aceptación del multiculturalismo, nosotros mostramos que cuando se trata de cederpoder a un colectivo indígena se produce el efecto contrario.

Desde el punto de vista de las implicancias prácticas, problematizamos respecto a la posibilidad de incidencia de los grupos pro-autodeterminación toda vez que tienden a ser jóvenes, indígenas y urbanos, perfil que tiende a no participar de los procesos electorales. Por lo mismo, los actores políticos tendrían muy pocos incentivos de preocuparse de esta agenda en la medidaque los grupos que favorecen la autodeterminación tienden a no votar. Finalmente, el trabajo hace un aporte al diferenciar entre la aceptación social a la diferencia, de la aceptación a la redistribución de poder en una sociedad.

\section{Debate teórico sobre multiculturalismo y autonomía}

Un importante cuerpo de la literatura ha puesto énfasis en el estudio de las actitudes sociales entre grupos étnicos en una comunidad(Lambert \& Taylor, 1988; Betts, 1999; Fetzer \& Soper, 2011). Aquí el eje central de las preguntas se asocian con identificar los determinantes de las actitudes positivas/negativas entre un grupo cultural o étnico dominante y otros grupos sociales subordinados sean estos pueblos indígenas, grupos religiosos o migrantes. El nivel socioeconómico, niveles de educación, el lugar de residencia, y la edad parecen ser variables muy relevantes que explicarían la aceptación/rechazo del denominado multiculturalismo. Por ejemplo, Lambert y Taylor (1988)encuentran que la clase trabajadora de sectores urbanos en Estados Unidos son menos tolerantes a políticas que promuevan el multiculturalismo en las escuelas. Otros estudios han destacado el nivel de educación como un fuerte predictor de actitudes favorables hacia la diversidad. Esto es, mientras mayor es el nivel de escolaridad, mayor es la aceptación de convivir en ambientes donde se respeten diferentes culturas (Betts, 1999; Markus, 2011).De acuerdo a Fetzer y Soper (2011), son los estudiantes universitarios los primeros en ser expuestos a nuevas ideas, realidades y tendencias lo cual acrecienta la posibilidad de que cambien sus 
valores por otros de índole más bien liberal, o menos conservador. Otros autores plantean que el pensamiento crítico, que por lo general es impulsado en los ambientes universitarios, lleva a las personas a dejar de lado sus prejuicios y estereotipos sociales a la vez que da cabida para identificarse con posiciones liberales. De acuerdo a esto, el incremento en el nivel educacional impulsaría el apoyo hacia los derechos de las minorías tanto sexuales como raciales y étnicas (Weakliem, 2002).

Otros trabajos llaman la atención del vínculo territorial y su impacto en temas relacionados con la diversidad cultural, multiculturalismo y derechos indígenas (Marks \& Mcdonell, 1996; Van Den Eynde \& Dharmalingam, 2008). En un estudio que mide la opinión pública en torno a los derechos aborígenes en Australia, Van Den Eynde y Dharmalingam (2008) encontraron importantes diferencias de acuerdo a la zona en que se vive. Quienes habitaban en zonas rurales muestran una clara tendencia a limitar los derechos de los aborígenes mientras que quienes habitan las ciudades abogan por ampliar el marco jurídico que los protege. De acuerdo a los autores esto podría deberse a la posibilidad de que quienes habitan en zonas rurales pueden sentirse perjudicados por una eventual política de devolución de tierras, lo cual afectaría directamente sus intereses materiales.

Asimismo, otros estudios advierten de la importancia de observar cómo ciertas condiciones políticas afectan en el mediano y largo plazo las actitudes sociales sobre aceptación del multiculturalismo.Por ejemplo, Ward y Masgoret(2008) muestran cómo en Canadá y Nueva Zelandia existe mayor aceptación social al multiculturalismo que en algunos países europeos, dando cuenta de los contextos históricos de políticas implementadas por los gobiernos, combinado con la experiencia política de negociaciones con pueblos indígenasque demarcaron el debate en esos países. En algunos países de Europa Occidental, las actitudes sociales menos favorables hacia el multiculturalismo se vincularían principalmente con olas migratorias recientes y a la ausencia de políticas estatales sobre el particular.

Pero hasta aquí la interrogante central se refiere a la aceptación o tolerancia respecto de otros grupos. Betts(1999) realiza una importante contribución al señalar que estos niveles de aceptación van a depender mucho de los recursos a distribuir en una sociedad. Así, el autor sugiere que podríamos hablar de un "multiculturalismo soft" y que alude a la tolerancia respecto de la existencia de otro grupo en la sociedad, y un "multiculturalismo hard" que se refiere a la aceptación que el Estado disponga de recursos para proteger y promover la diversidad cultural en una sociedad. En su estudio aplicado en Australia, Betts encontró que mientras 3/5 de las personas con educación superior favorecían el "multiculturalismo hard", solo 1/4 de las personas sin educación universitaria lo hacían (Betts, 1999). Más recientemente, Markus (2011) encuentra la misma tendencia en la variable educación en un estudio de opinión pública aplicado en el estado de Victoria (Australia), destacando además las variables de edad y nivel socioeconómico, esto es, que mientras más jóvenes y de mayor nivel socioeconómico mayor es la propensión a aceptar "multiculturalismo hard". 
Aquí asoma un debate más sustantivo sobre el modo en que se entiende el reconocimiento y la aceptación de un "otro" colectivo en la sociedad. Esto llevó a autores como Hale (2002) a referirse al “multiculturalismo neoliberal” que se asocia con la aceptación de la diversidad cultural en la sociedad. Pero esta tolerancia a lo diverso no implica necesariamente que se permita la emancipación de colectivos indígenas que se auto reconocenen un proyecto identitario diferente al promovido por un sistemacapitalista. Así, se produce una contradicción entre el reconocimiento de la diversidad y la amenaza de la autodeterminación (Ver Briones2007). Los grupos dominantes querrán siempre tomar la iniciativa de control cultural, social y político incluso si los grupos subalternos se rebelan (Modonesi, 2017).

En efecto, en el caso de los pueblos indígenas el debate sobre el reconocimiento alude simultáneamente a las relaciones interculturales y respecto de la distribución de poder político, económico y cultural dada la disputa histórica de territorios. Por lo mismo, cuando nos referimos a "autodeterminación” nos referimos al proceso de emancipación (Modonesi, 2010) que permitirá a estos actores poder autogobernarse colectivamente de acuerdo a las tradiciones que les son propias; el hecho que puedan administrar autónomamente un determinado territorio; y que desarrollen y respeten sus culturas y tradiciones, entre otras dimensiones (Al respecto ver Modonesi, 2010; Rosales, 2010;Marimán, 2012; Nájera, 2013) ${ }^{1}$. En este estudio nos interesa precisamente explorar la aceptación social de la autonomía indígena en el sentido indicado aquí de autodeterminación y no solo referida a la tolerancia al multiculturalismo.

Existen pocos estudios que aborden las actitudes sociales sobre procesos dedistribución de poder y otorgamiento de reconocimiento de derechos más sustantivos a ciertos grupos de la sociedad. Por ejemplo, González y Mackenna (2017) , encuentran que existe una relación positiva entre el mayor nivel de contactos de los indígenas (en este caso Mapuche en Chile) con no indígenas y la preferencia de la integración por sobre la autonomía de los pueblos indígenas. En el caso de los no indígenas el efecto de la interacción no produciría efectos en mayor o menor preferencia por la autonomía (González \& Mackenna, 2017). Asimismo, otros trabajos aplicados a contextos no latinoamericanos como España y Canadá nos permiten dar pistas teóricas y empíricas sobre este asunto. En el caso de España se han realizado estudios que examinanlas actitudes sociales en relación a los procesos de autonomía en el país Vasco, Cataluña y otras comunidades.En uno de los más completos estudios sobre el tema, Liñeira (2014)indica que las variables más significativas asociadas a la preferencia por autonomía son laauto identificacióncon un determinado grupo que históricamente ha reclamado más autonomía, la identificación política y la edad.

Lo interesante del trabajo de Liñeira es que permite un análisis de cohortes generacionales, advirtiendo que no sólo las generaciones más recientes son más sensibles a favorecer la autonomía, sino que además se 
advierte un cambio en la intensidad de aquella aceptación dentro de cada generación (Liñeira 2014,p.98). Lo propio sucede con la ideología, advirtiéndose que mientras en 1992 quienes se auto identificaban con la izquierda tendían a ser más autonomistas y los de derecha más centralistas, en el año 2002 sucedía precisamente lo contrario,y en el 2010 esta variable dejó de ser relevante dado que el autonomismo se muestra transversal a las ideologías (Liñeira 2014,p.117).

Por su parte, un estudio sobre las actitudes sociales de los canadienses respecto de la independencia de Quebec, demostró actitudes favorables hacia el independentismo entre aquellos simpatizantes del partido Quebequés, los jóvenes, aquellos con educación universitaria y de nivel socioeconómico alto (LeDuc, 1977), remarcando las mismas tendencias que se observan en otros contextos. Por otra parte, para el caso de Estados Unidos, se encontró que mientras mayor sea la percepción de opresión en las minorías, mayor será la tendencia a considerar al grupo mayoritario como una amenaza y más aún será el apoyo intragrupal (Bobo \& Hutchings, 1996).

En síntesis, el estudio de las actitudes sociales respecto del multiculturalismo ha especificado una serie de factores predictivos que incluyen educación, nivel socioeconómico, zona geográfica, edad y auto identificación con un grupo identitario (nación, etnia o pueblo indígena). Una distinción central de las actitudes respecto de la multiculturalidad se asocia con la distribución de recursos y de poder en la sociedad. Los estudios que han abordado las actitudes sociales sobre diversas autonomías(sean ellas de grupos indígenas o no) han destacado como factores determinantes a la ideología, la auto identificación con un grupo étnico determinado, la edad y el nivel de educación.

\section{Contexto histórico, político y social.}

Como vimos en el apartado anterior, entonces, la literatura ha insistido correctamente en indicar que las percepciones y actitudes sociales están mediadas por un contexto histórico, político y social. En América Latina en general y Chile en particular el debate sobre los pueblos indígenas se ve afectada por una extensa historia de usurpación, abusos y falta de reconocimiento. Desde el punto de vista histórico, el tema nos remota a modo en que la Corona Española y luego el Estado de Chile se relacionó con los pueblos indígenas que habitan el espacio territorial de lo que posteriormente se estableció como una república. Los españoles adquirieron un relativamente rápido control del territorio hasta el río Biobío. Entre fines de 1500s y la independencia de Chile en 1810 se produjo una constante situación de conflicto en la frontera sur de la Araucanía. La imposibilidad de ocupar dicho territorio dada la resistencia indígena llevó a la Corona española a realizar una serie de tratados y acuerdos ("Parlamentos") para contener el conflicto y definir la propiedad de las tierras (“Títulos de Merced”). Una vez producida la 
independencia, el gobierno de Chile, que tampoco podía iniciar una campaña militar en dicha zona, firmó un tratado con el pueblo mapuche (Tratado de Tapihue, 1825) que reiteró la existencia de una frontera en el rio Biobío(Correa \& Mella, 2010; Bengoa, 2014).

Las circunstancias cambiaron y a mediados del siglo XIX se inició un proceso de intensificación del conflicto en la frontera, colonización del extremo sur con colonos alemanes, y la ocupación del territorio de la Araucanía que terminó por controlar todo el territorio. A partir de entonces, se produjo una política del Estado de Chile de asimilación y construcción social de la "nación chilena” y que incluyó a una decena de pueblos indígenas que habitaban el territorio.

Durante el siglo XX se mantuvo esta visión asimilacioncita. Mientras las élites más conservadoras negaban la existencia de estos "pueblos” o "naciones” indígenas”, las élite políticas chilenas más críticas de izquierda asociaban la problemática indígena como uno de clase social, refiriéndose a ellos usualmente como campesinos pobres que demandaban tierras. Los indígenas rápidamente fueron asimilados ocupando el más bajo de los estatus sociales del territorio, subordinados a relaciones de dependencia con los grandes hacendados que pasaron a controlar progresivamente el territorio.

Desde el punto de vista social, existe particularmente en la cultura mapucheuna auto-percepción de un pueblo guerrero; que libró una batalla de más de 300 años en contra de la Corona Española y del Estado de Chile por sus territorios. Con alzas y bajas, durante la historia del siglo XIX y XX se recuentan una serie de episodios de resistencia respecto de la ocupación del Estado de Chile. Posteriormente durante la dictadura de Pinochet además de la represión ejercida sobre los pueblos indígenas, inició un proceso de privatización de las tierras ancestrales, terminando con la concepción comunitaria de la tierra (Decreto 2.568/ 1978). Cabe advertir que en la actualidad quienes se identifican como indígenas son poco más de 1,5 millón de habitantes que representan el 9\% de la población total. De ellos el 84\% dice pertenecer al pueblo mapuche. El 75,3\% de aquella población vive en zonas urbanas y el 24,7\% del total vive en la Región Metropolitana (CASEN, 2015).

Con la recuperación de la democracia, se inicia un nuevo ciclo político y social para los pueblos indígenas y para el pueblo mapuche en particular. Primero, el gobierno democrático se comprometió a realizar una serie de acciones políticas asociadas a su reconocimiento, firma de acuerdos internacionales sobre la materia, y actos reparatorios asociados a la devolución de tierras que comenzó a implementar tímidamente a partir de 1990.A fines de aquella década se inicia un nuevo ciclo de protestas Mapuche a propósito de la construcción de una represa en lo alto del Biobío. A la intensificación de proyectos económicos extractivistasde corte neoliberal y de uso intensivo de recursos naturales (forestales, pesca, construcción de hidroeléctricas), le siguió una dinámica social de radicalización y mayor activismo 
social de grupos indígenas que demandaban del Estado de Chile el respeto de acuerdos firmados a inicios del siglo XIX. Se creó la Coordinadora AraucoMalleco que inició acciones de recuperación territorial y acciones de protesta en contra de propiedades privadas que reclamaban suyas (Toledo, 2006).

La respuesta institucional del Estado de Chile ha sido pendular y variada. Desde el punto de vista institucional el Estado firmó el convenio 169 de la Organización Internacional del Trabajo (OIT) que establece el deber de consulta a los pueblos indígenas de asuntos que les impacten en forma directa (2009), y ha creado una serie de Comisiones y Planes para responder a través de políticas públicas a las demandas territoriales y de reconocimiento. ${ }^{2}$ Adicionalmente, se ha intensificado la represión policial en contra de los movimientos de protesta indígena, causando incluso víctimas civiles por violencia policial. Además, se ha respondido penalmente utilizando la ley antiterrorista para enfrentar la violencia en la zona lo que ha generado una serie de protestas nacionales e internacionales dados los cuestionamientos respecto del debido proceso que involucra dicho procedimiento.

Otros pueblos indígenas también han iniciado movilizaciones. Las más relevantes se asocian al pueblo Rapa Nui, quienes a través de acciones de protesta han ocupado espacios como un hotel privado en la isla, demandando mayor atención del Estado de Chile. Más recientemente, el pueblo Rapa Nui presentó una demanda ante la Comisión Interamericana de Derechos Humanos reclamando la propiedad colectiva de la Isla de Pascua así como espacios más relevantes de autonomía ${ }^{3}$. A lo anterior se suma una nueva generación de intelectuales y dirigentes sociales indígenas (principalmente Mapuche) que participan de debates públicos sobre identidad, reconocimiento y respecto de las demandas de los pueblos indígenas ${ }^{4}$.

Lo anterior se ha manifestado políticamente en el aumento en la cantidad de alcaldes que se auto reconocen como indígenas, el incremento sustantivo en el número de candidaturas al Congreso Nacional de dirigentes indígenas, y la solidaridad del voto hacia candidaturas indígenas(Gunderman, 2007; Contreras \& Morales, 2017). Desde el punto de vista de los partidos tradicionales, en otros trabajos hemos constatado la mayor relevancia que ha adquirido la temática indígena en las plataformas de las candidaturas presidenciales, y el progresivo énfasis en la necesidad de reconocer a los pueblos indígenas en tanto tales en la Constitución (Fuentes, s/f).

En síntesis, observamos un momento político y social de mayor visibilidad social y política de un conflicto que ha sido permanente, primero entre los pueblos indígenas y el Imperio Español y luego con el Estado de Chile. Esta mayor visibilidad se explica por un conjunto de transformaciones a escala global y también regional donde se reconfiguran las fuerzas y discursos en este campo de disputa. Lo ante- 
rior debiese implicar que existe cierta correspondencia entre este debate político y las percepciones sociales que es lo que nos proponemos analizar en lo que sigue. Siguiendo a la literatura revisada, debiésemos esperar una mayor preferencia por la autonomía entre aquellos que se identifican como indígenas, de sectores urbanos y jóvenes.

\section{Metodología}

En este trabajo analizamos las percepciones sociales respecto de la autodeterminación de los pueblos indígenas. Para ello utilizaremos la Estudio Longitudinal sobre las Relaciones Interculturales (ELRI) llevada a cabo por el Centro de Estudios Interculturales e Indígenas (CIIR) y cuyo objetivo es estudiar las diversas dimensiones de las relaciones interculturales a través del tiempo. La unidad de análisis del instrumento son individuos entre 18 y 60 años que residen en territorios con características interculturales, esto es, comunas con más de un 9\% de población indígena o con más de un 0,04\% del total nacional. El diseño de este criterio se realizó integrando datos de otras fuentes como Encuesta de Caracterización Socioeconómica Nacional 2013(CASEN),Censo $2002^{5}$. El trabajo de campo se llevó a cabo entre el 9 de octubre y el 13 de diciembre de 2016 en 120 comunas del país considerando 3.617 encuestados seleccionados de forma aleatoria. El muestreo fue probabilístico y multi-etápico considerando en una primera etapa regiones y comunas de acuerdo al diseño (criterio de interculturalidad) y en una segunda manzanas y hogares de acuerdo a una selección aleatoria

Para el análisis se utilizará un modelo de regresión logística multinomial. Este modelo permite ver la relación entre un conjunto de variables independientes, ya sean estas continuas o categóricas, con una variable dependiente nominal con más de dos categorías (Hosmer \& Lameshow, 2000). En este sentido, el modelo se presenta como una extensión multivariante de la regresión logística tradicional (Fernández \& San Martín , 2000) que nos permitirá medir el nivel de adhesión a la demanda de autonomía utilizando, como veremosen los siguientes apartados, tres categorías de agrupación.

\section{Composición Muestra}

La Tabla 1 a continuación detalla la composición general de la muestra de acuerdo a la frecuencia de variables sociodemográficas de interés para esta investigación. En la Tabla 2 se ilustra la composición de acuerdo a laauto identificación con pueblo indígena. 
Polis, Revista Latinoamericana, $N^{\circ}$ 49, 2018

Tabla 1 : Composición muestra

\begin{tabular}{|c|c|c|}
\hline Categoría & Frecuencia & $\%$ \\
\hline \multicolumn{3}{|l|}{ Sexo } \\
\hline Hombre & 1289 & 35,6 \\
\hline Muje & 2328 & 64,4 \\
\hline \multicolumn{3}{|l|}{ Zona } \\
\hline Zona Urbana & 2653 & 73,3 \\
\hline Zona Rural & 964 & 26,7 \\
\hline \multicolumn{3}{|c|}{ Nivel Educacional* } \\
\hline Básica & 1373 & 38,6 \\
\hline Media & 1662 & 46,8 \\
\hline Superior & 518 & 14,6 \\
\hline \multicolumn{3}{|c|}{ Identificación Pueblo Indígena** } \\
\hline Indigena & 1827 & 50,9 \\
\hline No indigena & 1761 & 49,1 \\
\hline \multicolumn{3}{|l|}{ Macrozona } \\
\hline Zona Norte & 1017 & 28,1 \\
\hline Zona Centro & 951 & 26,3 \\
\hline Zona Sur & 1649 & 45,6 \\
\hline \multicolumn{3}{|c|}{ identificación Política ${ }^{* * *}$} \\
\hline Izquierda & 540 & 14,9 \\
\hline Centro & 538 & 14,9 \\
\hline Derecha & 366 & 10,1 \\
\hline Ninguna & 1881 & 52 \\
\hline
\end{tabular}

*64 valores perdidos NS/NR; **29 valores perdidos NS/ $\mathrm{NR} ; * * * 292$ valores perdidos NS/NR

Fuente: Elaboración propia en base a datos de ELRI 


\section{Tabla 2: Caracterización muestra por identificación Pueblo Indígena*}

\begin{tabular}{|c|c|c|c|}
\hline & & \multicolumn{2}{|c|}{ Auto identificación Pueblo Indígena } \\
\hline & & Indigena & No indigena \\
\hline \multirow[t]{2}{*}{ Sexo } & Mujer & 64,59 & 63,88 \\
\hline & Hombre & 35,41 & 36,12 \\
\hline \multirow[t]{2}{*}{ Zona } & Urbano & 72,09 & 74,45 \\
\hline & Rural & 27,91 & 25,55 \\
\hline \multirow[t]{3}{*}{ Macrozona } & Norte & 27,81 & 28,22 \\
\hline & Centro & 25,56 & 26,92 \\
\hline & Sur & 46,63 & 44,86 \\
\hline \multirow[t]{3}{*}{$\begin{array}{l}\text { Nivel } \\
\text { Educacional }\end{array}$} & $\begin{array}{l}\text { Básica o } \\
\text { menos }\end{array}$ & 41,44 & 35,80 \\
\hline & Media & 44,84 & 48,73 \\
\hline & Superior & 13,72 & 15,47 \\
\hline \multirow{4}{*}{$\begin{array}{l}\text { Posición } \\
\text { Política }\end{array}$} & Izquierda & 16,51 & 15,85 \\
\hline & Centro & 15,32 & 17,03 \\
\hline & Derecha & 9,92 & 12,14 \\
\hline & Ninguna & 58,25 & 54,98 \\
\hline
\end{tabular}

* Considera los datos perdidos de tabla 1

Fuente: Elaboración propia en base a datos de ELRI

\section{Variable dependiente: autonomía}

La autodeterminación es un derecho establecido por las comunidades políticas, y más recientemente en tratados internacionales, para definir el modo en que se auto-gobernarán.Tal como se señaló en la discusión teórica precedente, la autodeterminación alude al proceso de emancipación que, en el caso de los pueblos indígenas, presupone no tan solo un reconocimiento genérico por parte de la sociedad dominante, sino que una serie de condiciones materiales y subjetivas que a un determinado colectivo le permite auto gestionarse. Ghai (2000), por ejemplo, define la autonomía como“un dispositivo que permite a los grupos étnicos u otros que adscriban a una identidad distinta el ejercicio de control directo de sus propias necesidades y preocupaciones mientras relega a la entidad más grande cubrir esos poderes que garantizan los intereses comunes”6 (Ghai, 2000,p. 8). La definición que plantea este autor pone el acento en la dimensión política del concepto, haciendo referencia al derecho de los pueblos con características étnicas particulares a poder decidir, en base a sus propias necesidades y de manera autónoma, sobre su propio porvenir.Por su parte, Kuppe (2010), asocia el concepto de autonomía a "ciertos arreglos que se aplican en un ámbito geográfico o territorial particular dentro de la estructura de organización 
territorial de un Estado.” (p. 109). Agrega después que dichos arreglos tienen su fundamento en las características culturales específicas de la población correspondiente, por lo que la condición de aplicación es la existencia de áreas territoriales, delimitables e identificables, de asentamientos de los grupos que van a ser los beneficiarios de dichos arreglos.

Hannikainen (2013), inspirado en el caso de Finlandiaplantea que la autonomía, como una manera de llevar a la práctica el derecho de la autodeterminación, requiere de la creación de tres órganos relevantes en los territorios autónomos: una asamblea legislativa (parlamento local) que represente al pueblo autónomo en sus propios asuntos, un poder ejecutivo (gobierno local) que se forme de acuerdo a los principios democráticos y que cuente con la aprobación de la asamblea legislativa. En otro texto, el mismo autor abre la posibilidad a que esta delegación se exprese de diferentes maneras, como lo puede ser a través de representación parlamentaria en forma de escaños reservados, con lo que se plantea otra dimensión política de la autonomía que hace referencia a la representación de los pueblos bajo este estatuto en la toma de decisiones del Estado mayor(Hannikainen, 1998).

De acuerdo a lo señalado en la literatura revisada, consideraremos en nuestra metodología tres dimensiones del derecho a la autodeterminación y que se expresan en una dimensión territorial vinculadocon la devolución de tierras, otra de la administración autónoma de ciertos territorios, y una tercera de representación de los pueblos a través de escaños reservados en el Congreso Nacional.Mientras las dos primeras dimensiones son manifestaciones de una emancipación efectiva (control político y control territorial), consideramos que la última dimensión es relevante porque implica la aceptación o reconocimiento que los pueblos indígenas tienen el inherente derecho a tener un espacio de representación propio en la principal institución del Estado-nacional.

Para operacionalizar estas tres dimensiones se ha construido una variable dependiente llamada "autonomía”, la cual posee tres categorías; 1=Autonomista, $2=$ Indefinido, 3=No autonomista. Para construir esta variable se seleccionaron tres preguntas del módulo $\mathrm{E}$ de la encuesta que según nuestro planteamiento, y vinculado a la teoría sobre derechos de autonomía, en su conjunto logran medir esta variable latente de adhesión a la demanda autonomista, a saber, la dimensión territorial, la de autogobierno político, y la de representación. Estas preguntas responden a cada una de las dimensionesque desde la teoría se proponen. Las preguntas seleccionadas miden el grado de acuerdo con algunas políticas públicas, la escala de respuesta recodificada va de 1 a 3 donde $1=$ Desacuerdo, $2=\mathrm{Ni}$ de acuerdo ni en desacuerdo y $3=$ De acuerdo. La tabla 2 a continuación muestra un panorama descriptivo de las variables seleccionadas 
Tabla 3. Nivel de acuerdo dimensiones autonomía

\begin{tabular}{llr}
\hline \hline Variable & Pregunta & \% Acuerdo \\
\hline E1_1 & Devolver tierras a indigenas (Dimensión territorial) & 70,8 \\
E1_4 & $\begin{array}{l}\text { Permitir que algunos territorios sean administrados por comunidades indígenas } \\
\text { (Dimensión política de autogobierno) }\end{array}$ & 62,7 \\
E3_4 & $\begin{array}{l}\text { Debiese existir cupos especiales reservados para indígenas en el congreso } \\
\text { (Dimensión política de representación) }\end{array}$ & 59,0 \\
\hline \hline
\end{tabular}

Fuente: Elaboración propia basado en datos ELRI

Posterior a la selección de las variables, se generan contadores del número de veces que cada persona marcó "De acuerdo", "Ni de acuerdo ni en desacuerdo" (NN) y "En desacuerdo" para cada una de las tres preguntas. En base a las posibles combinaciones de respuestas se crea la variable "Autonomismo" y sus categorías. La distribución de casos para esta variable se muestra en la tabla 2.

Tabla 4. Descriptivos variable Autonomía

\begin{tabular}{|c|c|c|c|c|c|}
\hline & & Frecuencia & Porcentaje & Porcentaje válido & Porcentaje acumulado \\
\hline \multirow[t]{4}{*}{ Válido } & Autonomista & 2096 & 57,9 & 60,2 & 60,2 \\
\hline & Indefinido & 931 & 25,7 & 26,7 & 86,9 \\
\hline & No autonomista & 456 & 12,6 & 13,1 & 100,0 \\
\hline & Total & 3483 & 96,3 & 100,0 & \\
\hline Perdidos & Sistema & 134 & 3,7 & & \\
\hline Total & & 3617 & 100,0 & & \\
\hline
\end{tabular}

Fuente: Elaboración propia

\section{Variables independientes}

Las variables independientes se dividen en dos categorías: una que representan el conjunto de variables sociodemográficas de los individuos tal como sexo, edad, zona de residencia (urbano/rural), nivel socioeconómico, nivel educacional, macrozona e identificación con pueblo indígena(indígena/ no indígena). Y una segunda categoría de factores subjetivos como la identificación política. Estas variables responden a la descripción realizada en el apartado sobre determinantes de la demanda autonomista. 
Nuestras hipótesis son las siguientes:

\section{Autoidenficación étnica}

Auto identificarse con un pueblo indígena se correlaciona positivamente con favorecer políticas de autonomía indígena.Tal como indicábamos previamente, los estudios muestran sostenidamente que aquellas personas que se auto identifican con determinadas identidades sociales (étnicas o "nacionales") tienden a favorecer la autonomía toda vez que se percibe como una retribución por un daño causado y que usualmente está asociado a la devolución de ciertos derechos ancestrales territoriales y de capacidad de autogobierno.

\section{Urbano/rural}

$\mathrm{Al}$ respecto, hipotetizamos que quienes residen en sectores urbanos muestran una mayor predisposición a favorecer políticas de autonomía indígena que aquellos que residen en zonas rurales. Esto debido a que en sectores urbanos se advierte una mayor socialización y politización del tema. En sectores rurales tienden a predominar relaciones verticales y de dependencia, lo que lleva a los individuos a sostener posiciones más conservadoras.

\section{Escolaridad}

Los estudios comparados han mostrado consistentemente que a mayor nivel de escolaridad, mayor es la propensión a favorecer el multiculturalismo. Sin embargo, en nuestro caso sostenemos que se debería dar una relación inversa, es decir, debiesen ser los sectores de menor escolaridad los que favorezcan la autonomía y los de mayor escolaridad los que más la rechaza.

Esto ocurre porque en el caso de Chile el nivel de escolaridad está fuertemente correlacionado con el nivel socioeconómico. Dado que el debate sobre la autonomía se refiere a la redistribución de poder político y económico (tierras), entonces debiésemos esperar que las personas con mayor escolaridad $-\mathrm{y}$ por ende, de posiciones altas de la escala socialse mostrasen contrarios a ceder un recurso económico tan vital como es la tierra.

\section{Autoidentificación política}

El eje izquierda-derecha debiese ordenar las preferencias en torno de la autonomía, asumiendo que las personas que se auto identifican con la izquierda son más favorable a la autonomía y mientras que los de derecha menos favorables.

Como los sectores políticos de la derecha tradicional han defendido los intereses de los hacendados, y los de la centro-izquierda los intereses de los campesinos-pobres en el caso de Chile, es esperable 
que exista algún tipo de vínculo entre auto identificación política y predisposición a favorecer/rechazar la autonomía de los pueblos indígenas. Desde el punto de vista político-ideológico, otros estudios muestran que los partidos políticos más de izquierda efectivamente tienen una mayor predisposición a favorecer políticas de autonomía, mientras los partidos más de derecha han tradicionalmente tendido a negar el reconocimiento, o favorecer un reconocimiento tenue de derechos (Fuentes, s/f). En concordancia con lo anterior, Martínez (2009) plantea que durante la década de 1980 y 1990 los mapuches ${ }^{7}$ han militado en organizaciones políticas de centro e izquierda y que fueron actores activos en el proceso de recuperación de la democracia. Sin embargo, también es relevante señalar que la coalición de centro-izquierda (Concertación) en tanto coalición programática hasta hace muy poco ha tenido posturas ambiguas asociadas al reconocimiento. Asimismo, el vínculo cotidiano entre electores y representantes no siempre está cruzado por una determinada ideología, sino que por el tipo de transacciones que se realizan entre representantes y representados (De Cea \& Fuentes, 2016).

\section{Sexo}

De acuerdo a lo descrito por la literatura (Sirlopú, Melipillán, Sánchez, \& Valdés, 2015), la variable sexo ha presentado efectos poco intensos en los estudios sobre multiculturalismo y derechos indígenas por lo que no se espera una incidencia significativa de esta variable en el modelo (Sirlopú, Melipillán, Sánchez, \& Valdés, 2015). No obstante aquello, de mediar algún efecto, se espera que sean las mujeres quienes sean más proclives a la demanda autonomista indígena. Estudios realizados en Estados Unidos sostienen que son ellas quienes se muestran más proclives a las ideas liberales en cuanto a políticas raciales (Steeh \& Schuman, 1992; Shapiro \& Mahajan, 1986). Otros estudios demuestran que son las mujeres quienes apoyan en mayor medida la diversidad cultural por sobre los hombres y son más proclives a generar lazos con grupos étnicos diferentes (Kirkpatrick Johnson \& Mooney Marini, 1998).Analizando el caso chileno, Sirlopú et al. (2015)encuentran una mayor propensión de las mujeres de aceptar la multiculturalidad.

\section{Edad}

Tal como otros estudios han mostrado, sostenemos que mientras más jóvenes las personas, mayor es la propensión a favorecer políticas de autonomía indígena.Ronald Inglehart (1991) identifica dos conjuntos de valores a los cuales las personas adscriben y en torno a los cuales se pueden agrupar: los valores materialistas y post materialistas. Dentro de los primeros se encuentran las necesidades fisiológicas de seguridad física y económica mientras que en los segundos se encuentran todas aquellas necesidades sociales y de actualización como la estética, lo intelectual y el sentido de pertenencia. Dentro de las segundas el autor identifica las necesidades de libre expresión, de la mejor distribución tanto económica como de poder político y la importancia de las ideas. 
Así, en los países post industriales de occidente se ha producido un cambio intergeneracional de valores materialistas hacia valores postmaterialistas, siendo los jóvenes quienes dan mayor importancia a estos últimos mientras que en las personas de más edad prevalecen valores materialistas. De acuerdo a la teoría de la socialización y la escasez del mismo autor, los valores se formarían durante la adolescencia y los primeros años de la adultez. Aplicado a nuestro caso de estudio esto nos permitiría predecir que encontraremos un efecto importante de la edad como determinante de la adhesión a la demanda autonomista, tomando en cuenta que el cohorte etario joven es aquel que creció post dictadura, en una época de seguridad material y física sin precedentes en este país (Schmidt-Hebbel, 2006) por lo que esperamos encontrar mayor inclinación por valores postmaterialistas entre quienes tengan menos edad y, por ende, más chances de adherir a dicha demanda.Lo anterior es consistente con lo encontrado para el caso de Chile por Sirlopú et al. (2015),quienes encuentran una mayor predisposición de los jóvenes a favorecer políticas multiculturales que las personas de mayor edad.

\section{Macrozona}

Desde el punto de vista geográfico, la principal zona de conflicto se encuentra en áreas rurales del sur del país (región de la Araucanía) donde se han registrado atentados incendiarios, acciones de recuperación territorial y protestas de comunidades indígenas. Por tratarse de una región eminentemente inter-cultural donde conviven indígenas y no indígenas, esperamos encontrar que macrozonas alejadas del conflicto debiesen favorecer políticas de autonomía, mientras los que residen en zonas de conflicto, debiesen rechazarla por la proximidad de los incidentes.

\section{Resultados}

La tabla 3 muestra los resultados de los modelos de regresión logística multinomial realizados. En primer lugar, se advierte que un determinante significativo, aunque marginal, para la demanda autonomista es el sexo. Acá encontramos, en concordancia con lo descrito en nuestras hipótesis, que quienes adhieren a la demanda autonomista indígena tienden a ser mujeres, mientras que por otro lado los hombres tienden a ser no autonomistas $(b=.197, \mathrm{p}<.90)$. Una segunda variable sociodemográfica que se presenta como significativa es la edad. En concordancia con lo que sugerían nuestras hipótesis, la edad se muestra como un factor significativo en la adhesión a la demanda autonomista. El modelo indica que a menor edad más chances habrá de adherir a la demanda autonomista, tanto con respecto a los indefinidos como con los no autonomistas $(b=.009, \mathrm{p}<.99$ para ambos casos).

Con respecto a la dimensión urbano-rural, podemos ver que la 
variable de referencia autonomía marca una tendencia positiva tanto con respecto a indefinidos como no autonomistas. Sin embargo, sólo para el caso de no autonomista tenemos una relación significativa (b = .641, p <.99). Estos datos confirman lo que otros estudios han señalado referido a que quienes habitan en zonas urbanas tendrán más posibilidades de adherir a la demanda autonomista que quienes habitan en zonas rurales.

Un hallazgo importante a discutir en este estudio tiene que ver con el nivel educacional. De acuerdo a investigaciones previas aplicadas en otros contextos, el mayor nivel educacional incrementa las probabilidades de adherir a demandas favorables a los derechos de los grupos minoritarios. En cambio, nuestro modelo arroja como resultado que quienes tienen un nivel educacional básico son más favorables a la demanda de autonomía que el resto de las categorías ( $b=-.309$, p $<0,99$ con respecto a ser indefinido). En la misma línea, un factor que destaca tiene que ver con la tendencia significativa de las personas que poseen educación superior a rechazar la demanda autonomista $(\mathrm{b}=, 421 \mathrm{p}<$ 0,95). Lo anterior podría deberse a lo que indicábamos en nuestra hipótesis referido a que en el caso de Chile el nivel de escolaridad se asocia con el nivel socioeconómico. Como la demanda por autonomía se asocia con la redistribución de poder político y económico, entonces resulta esperable que sean los sectores de escolaridad baja los que la favorecen y los sectores de escolaridad alta los que la rechacen.

Al considerar el comportamiento por macrozonas, vemos que quienes viven en la macrozona norte y zona central del país se muestran más bien favorables a la autonomía en relación a las personas de la macrozona sur (coeficientes negativos y significativos a un 99\% para todos los casos). Este es un resultado relevante a analizar en la medida que sugiere que la demanda autonomista no se encuentra territorializada geográficamente en la zona donde se concentra el conflicto. Este elemento será discutido en las conclusiones finales.

Finalmente, en relación a la identificación ideológica, el modelo se comporta de acuerdo a lo esperado existiendo una relación significativa con nuestra variable autonomía en los dos extremos del espectro; esto quiere decir que mientras los encuestados que se autoidentifican con la izquierda se muestran favorables a la autonomía tanto con respecto a ser indefinido como a ser no autonomista $(b=-.256$ y $b=-.364$ respectivamente; $\mathrm{p}<0,95$ para ambos casos), quienes se identifican con la derecha se muestran contrarios a ella. 
Polis, Revista Latinoamericana, $N^{\circ}$ 49, 2018

Tabla 5. Modelos de regresión logística multinomial para explicar las determinantes del autonomismo

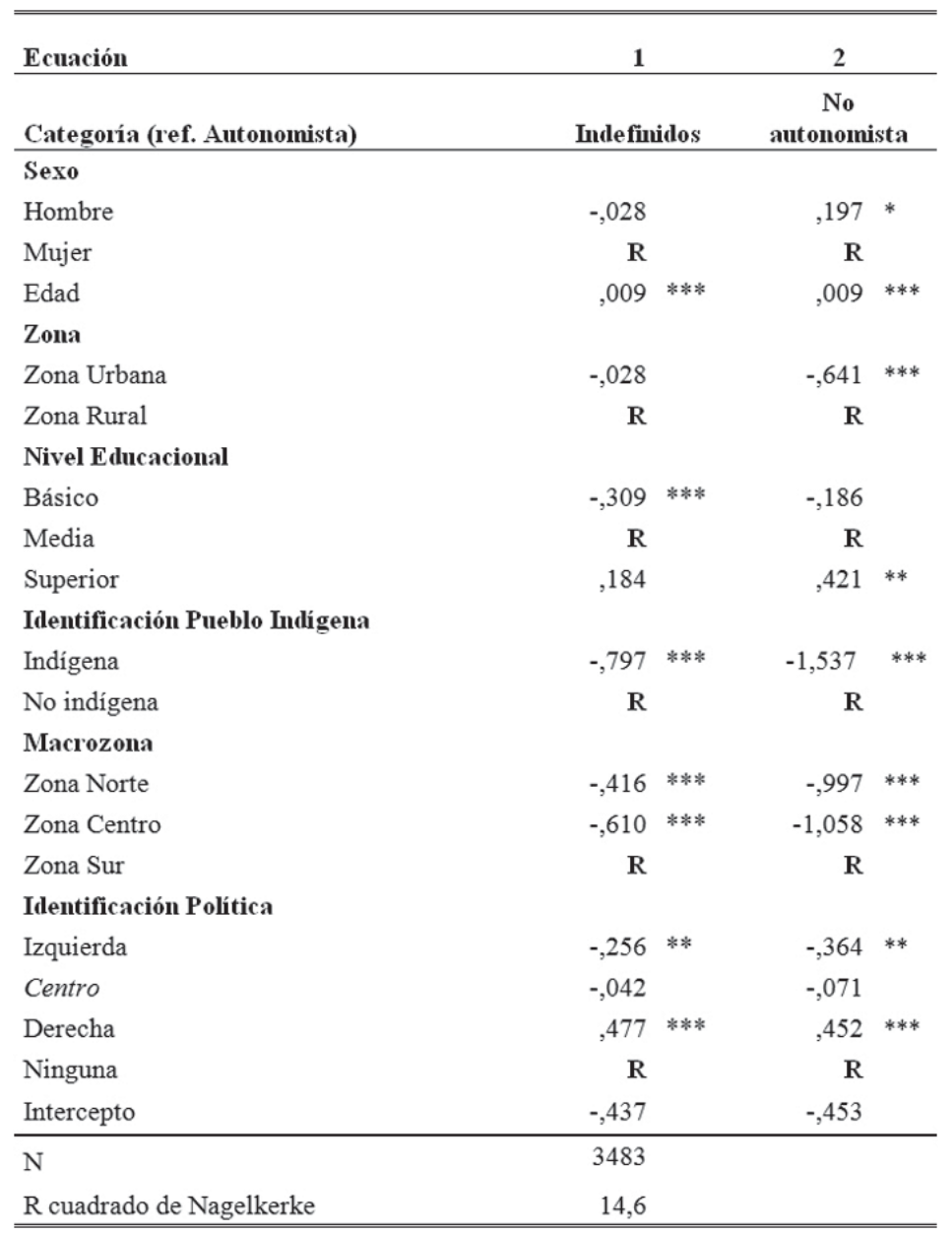

*** $\mathrm{p}<0,01 ; * * \mathrm{p}<0,05 ;{ }^{*} \mathrm{p}<0,1$

Fuente: Elaboración Propia 


\section{Discusión}

$\mathrm{Al}$ analizar la demanda por autonomía operacionalizada aquí en términos de la administración autónoma de territorios, autogobierno y representación de los pueblos en el Congreso, observamos que ella tiende a ser más relevante entre personas que se auto identifican como indígenas, jóvenes, con niveles de escolaridad baja, que viven en zonas urbanas, de izquierda, y de la macrozona centro y norte del país.

En términos generales, lo anterior es muy consistente con lo que otros estudios han demostrado sobre las actitudes sociales hacia grupos que demandan mayores niveles de autonomía. Sin embargo, un aspecto que merece atención es la variable escolaridad ya que nuestro estudio contradice lo que la literatura ha señalado por cuanto es esperable observar una asociación entre mayor escolaridad y mayor demanda por autonomía y aquello no sucede en este caso. Esto podría deberse a la asociación que existe en Chile entre alta escolaridad y mayores niveles socioeconómicos. Como la demanda por autonomía afecta directamente los intereses económicos de las élites, es probable que las personas de niveles de educación alta asocien esta demanda con un riesgo a sus intereses de clase social.Ello advierte de una importante consecuencia de política dada la alta concentración de poder político y económico en el país. En otras palabras, cualquier opción de colocar en la agenda de discusión mayores grados de autonomía-y con esto, mayores grados de redistribución de poder político, cultural y de recursos territoriales - muy probablemente serán resistidos por los grupos altos de la sociedad. Por otra parte, seguramente en los estratos bajos es más intenso el apoyo a la autonomía porque probablemente se asocia con políticas redistributivas que eventualmente podrían beneficiarlos.

Nuestro estudio confirma además varias intuiciones que se relacionan con el movimiento social indígena. En primer lugar, es plausible que dicho movimiento adquiera mayor radicalidad en sus demandas por autonomía en los centros urbanos más que en las zonas rurales del país. Asimismo, también es esperable que esta demanda sea más popular entre los jóvenes que entre personas de mayor edad. Como además la población indígena radica principalmente en ciudades, es precisamente allí donde es plausible que se articulen demandas crecientes por autonomía. Lo anterior podría deberse también a la intensidad del modelo neoliberal extractivista en zonas rurales, que tiende a reproducir relaciones de subordinación cuestión que se hace menos intensa en las ciudades.

Lo anterior tiene importantes y paradojales consecuencias para el sistema político. Por ejemplo, sabemos que la mayoría de los partidos políticos basa su apoyo electoral en sectores sociales medios y medios-altos, y personas mayores de 45 años. Asimismo, algunos partidos más tradicionales de derecha y algunos más moderados tienen una fuerte penetración electoral en regiones y en zonas rurales. Lo anterior implica que existiría una desconexión entre el perfil de la base social de los partidos tradicionales 
(mayores de edad, sectores medio-altos en zonas urbanas, sectores populares en zonas rurales) y el perfil de aquellos que demandan mayores grados de autonomía (indígenas, jóvenes, urbanos, de sectores bajos). Así, es muy poco probable que la demanda por mayor autonomía sea canalizada y viabilizada a través de partidos tradicionales de todo el espectro político.Eventualmente, la emergencia de partidos nuevos que incorporan grupos etarios jóvenes podrían incluir este tipo de demandas, tal como se confirmó en la elección presidencial de 2017.

Una segunda paradoja es que parece ser que la proximidad con el conflicto mapuche en el sur de Chile es un inhibidor de la demanda por autonomía y no un catalizador de ella. Quienes favorecen mayores niveles de autonomía son quienes se encuentran fuera de la región que es objeto de demandas territoriales, por lo que podríamos indicar que es una “demanda desterritorializada”.

Nuestro trabajo deja, sin embargo, abierta una serie de problemáticas teóricas. La primera y más relevante es el significado que la sociedad le asigna al concepto de “autonomía”. En nuestro estudio operacionalizamos el concepto a partir de tres dimensiones muy acotadas y específicas. Sabemos que el concepto de "autodeterminación” no se agota en aquellas dimensiones, pero además, la literatura ha destacado que los conceptos de “nación”, “autodeterminación”, “autonomía” adquieren significados muy disímiles desde un punto de vista experiencial (Grimson, 2003). Se requiere complementar con trabajos cualitativos que indaguen en preguntas como: ¿cuál es el imaginario de la “autodeterminación” en la sociedad? ¿Se asocia sólo y exclusivamente a la redistribución de poder o también se vincula con dimensiones intersubjetivas de deseos de emancipación cultural? ¿Cómo se vinculan los procesos de individuación que promueve el modelo neoliberal y la preferencia por “autonomía colectiva”?

Futuros estudios podrían explorar también algunas de las determinantes de la autonomía. Por ejemplo, se requiere estudiar con mayor detenimiento el perfil de quienes son favorables a la autonomía analizando hasta qué punto el contacto intercultural (entre indígenas y no indígenas) favorecería el tener posiciones más favorables al autonomismo. Adicionalmente, podría explorarse la relación que existe entre los indígenas urbanos (y sus lazos más o menos recientes con los territorios) y su proclividad a favorecer mayores niveles de autonomía. Con todo, este trabajo entrega una primera aproximación relevante de los principales determinantes de las actitudes sociales hacia la autonomía territorial indígena en Chile, un tema crucial en la agenda de construcción de una ciudadanía que se acepta como pluriétnica y plurinacional. 


\section{Agradecimientos}

Agradecemos al Centro de Estudios Interculturales e Indígenas (CIIR) por el soporte brindado a la realización de esta investigación, así como también por el acceso a los datos del Estudio Longitudinal sobre Relaciones Interculturales (ELRI). El artículo es parte del proyecto FONDECYT 1170025 cuyo investigador responsable es Claudio Fuentes S. Los autores agradecen los útiles comentarios anónimos de los revisores de este artículo. 
Polis, Revista Latinoamericana, $N^{\circ}$ 49, 2018

\section{Notas}

${ }^{1}$ El concepto autodeterminación ha generado un extenso debate intelectual y político. Un trabajo particularmente relevante para el caso de Chile es el de José Marimán quien se explaya en las distintas variantes del autonomismo Mapuche y sus manifestaciones políticas (2012). Ver también Bengoa (2007).

${ }^{2}$ Entre otras instancias, se pueden mencionar la Comisión de Verdad y Nuevo Trato del presidente Ricardo Lagos (2003), el Plan Araucanía del presidente Sebastián Piñera (2012), Comisión Asesora Presidencial de la Araucanía de Michelle Bachelet (2016), y Plan Araucanía del mismo gobierno (2017).

${ }^{3}$ Ver "El nuevo conflicto entre el Estado de Chile y el pueblo Rapa Nui", La Tercera, 24 de septiembre, 2017.

${ }^{4} \mathrm{Al}$ respecto, pueden mencionarse por ejemplo el Centro de Documentación Mapuche ÑukeMapu, la Comunidad de historiadores Mapuche, Un análisis al respecto en Canales(2015).

${ }^{5}$ Para la auto identificación indígena, la encuesta utilizada emula la pregunta realizada en encuesta CASEN 2013 cuyo rango de respuesta varía entre no pertenecer a ningún pueblo o a alguno de los 9 reconocidos por la Ley 19.253. Sabemos que esta forma de medir el asunto en cuestión es controversial, sin embargo, hasta el momento es el más aceptado y utilizado. Para mayor información sobre medición de población indígena y correlato con identidad revisar Gunderman et. Al. (2005)

${ }^{6}$ Traducción propia.

${ }^{7}$ Pueblo indígena más numeroso de acuerdo a CASEN 2015. 


\section{Bibliografía}

Bengoa, J. (2007). La emergencia indígena en América Latina. Ciudad de México, México D.F: Fondo de Cultura Económica.

Bengoa, J. (2014). Historia del pueblo mapuche Siglo XIX y XX.Santiago; Chile: Editorial LOM.

Betts, K. (1999). The Great Divide: Inmigration politics in Australia. Sydney, Australia: Duffy and Snellgrove.

Bobo, L., \& Hutchings, V. (1996). Perceptions of Racial Group Competition: Extending Blumer's Theory of Group Position to a Multiracial Social Context. American Sociological Review, 61(6), 951-972.

Briones, C. et al. (2007). Escenas del multiculturalismo neoliberal. En: A. Grimson, Cultura y Neoliberalismo. Buenos Aires, Argentina: CLACSO.

Canales, P. (Julio de 2015). Historiografía Mapuche: Balances y Perspectivas de discusión en el Chile reciente. Conversación con Jorge Pinto Rodriguez. Izquierdas(24).

CASEN. (2015). Encuesta de Caracterización Socioeconómica Nacional. Santiago, Chile: Ministerio de Desarrollo Social.

Contreras, G., \& Morales, M. (2017). Ethnic solidarity and the vote: Mapuche candidates and voters in Chile. Journal of Ethnic and Migration Studies, DOI: 10.1080/1369183X.2017.1371582, 1-22.

Correa, M., \& Mella, E. (2010). Las razones del Illkun/Enojo. Santiago, Chile: LOM.

De Cea, M., \& Fuentes, C. (2016). Vendiendo soluciones. Campañas políticas tradicionales y profesionalizadas en Chile. Revista Internacional de Sociología, 74(3), 1-13.

Díaz Polanco, H. (2006), Elogio de la diversidad: globalización, multiculturalismo y etnofagia. Ciudad de México, México D.F.: Siglo XXI.

Dinerstein, A.et. al. (2013)Movimientos sociales y autonomía colectiva: la política de la esperanza en América Latina.Buenos Aires, Argentina:Capital Intelectual.

Fernández, V., \& San Martín , R. (2000). Regresión Logística Multinomial. Cuadernos de la Sociedad Española de Ciencias Forestales(18), 323327. 
Fetzer, J., \& Soper, J. (July de 2011). The Determinants of Public Attitudes toward the Rights of Indigenous Peoples in Taiwan. Taiwan Journal of Democracy, 7(1), 95-114.

Fuentes, C. (s/f). Divididos en el reconocimiento de los derechos de pueblos indígenas: Manifiestos programáticos en Chile (1989-2013).Inédito.

Ghai, Y. (2000). Ethnicity and Autonomy: A Framework for Analysis. En Y. Ghai (Ed.), Autonomy and Ethnicity. Negotiating Competing Claims in Multi-Ethnic States (págs. 1 - 20).

González, R., \& Mackenna, B. (2017). Relaciones Interculturales entre mapuches y no mapuches: desigualdad, segregación y autonomía. En I. Aninat, V. Figueroa, \& R. González (Edits.), El Pueblo Mapuche en el siglo XXI. Propuestas para un nuevo entendimiento entre culturas en Chile. Santiago, Chile: Colección Centro de Estudios Públicos.

Grimson, A. (2003). La nación después del (de) constructivismo. Nueva Sociedad, 184, 33-45.

Gunderman, H. (2007). Municipios y pueblos indígenas en Chile. En W. Assies, \& H. Gunderman (Edits.), Movimientos Indígenas y Movimientos locales en América Latina. Antofagasta, Chile: Universidad Católica del Norte.

Gunderman, H., Vergara del S, J., \& Foerster, R. (2005). Contar a los indígenas en Chile. Autoadscripción étnica en la experiencia censal de 1992 y 2002. Estudios Atacameños(30), 91-115.

Hale, C. (2002). Does Multiculturalism Menace? Governance, Cultural Rights and the Politics of Identity in Guatemala. Journal of Latin American Studies, 34 (3): 485-524.

Hannikainen, L. (1998). Self-Determination and Autonomy in International Law. En M. Suksi (Ed.), Autonomy: Aplications and Implications (págs. 79-94). Kluwer Law International.

Hannikainen, L. (2013). La Autonomía en Finlandia: la autonomía territorial de las islas Aland y la autonomía cultural del pueblo indígena saami. Revista d'estudis autonòmics i federals(17), 71-106.

Hosmer, D., \& Lameshow, S. (2000). Applied Logistic Regression . United States: Wiley Series in Probability and Statistics.

Inglehart, R. (1991). El cambio cultural en las sociedades industriales avanzadas.Madrid, España: CIS. 
Kirkpatrick Johnson, M., \& Mooney Marini, M. (1998). Bridging the Racial Divide in the United States: The Effect of Gender. Social Psychology Quarterly, 61(3), 247-258.

Kuppe, R. (2010). Autonomía de los pueblos indígenas - La perspectiva desde la Declaración sobre los derechos de los pueblos indígenas. En M. Gonzáles, A. Burguete Cal y Mayor, \& P. Ortiz-T (Edits.), La Autonomía a debate. Autogobierno indígena y Estado Plurinacional en América Latina (págs. 95-145). Quito, Ecuador: FLACSO.

Lambert, W., \& Taylor, D. (1988). Assimilation versus Multiculturalism: The views of Urban Americans. Sociological Forum, 3(1), 72-88.

LeDuc, L. (1977). Canadian Attitudes Toward Quebec Independence. The Public Opinion Quarterly, 41(3), 347-355.

Liñeira, R. (2014). El Estado de las autonomías en la opinión pública. Madrid, España: Centro de Investigaciones Sociológicas.

Marks, G., \& Mcdonell, P. (1996). New Politics? The Mabo debate and Public Opinion on Native Title in Australia. International Journal of Public Opinion Research, 8(1), 31-50.

Markus, A. (2011). Attitudes to Multiculturalism and Cultural Diversity. En M. Clyne, \& J. Jupp (Edits.), Multiculturalism and Integration. Australia: ANU Press.

Marimán, J. (2012). Autodeterminación. Ideas políticas mapuche en el albor del siglo XXI. Santiago, Chile: LOM Ediciones.

Martinez Neira, C. (Agosto de 2009). Transición a la democracia, militancia y proyecto étnico. La fundación de la organización mapuche Consejo de Todas las Tierras (1978-1990). Estudios Sociológicos, 27(80), 596-618.

Modonesi, M. (2010).Subalternidad, antagonismo, autonomía. Marxismos y subjetivación política. En:Cuadernos del pensamiento crítico LatinoamericanoN $\mathrm{N}^{\circ}$ 34, CLACSO.

Modonesi, M. (2017). Revoluciones pasivas en América Latina. Ciudad de México, México: Universidad Autónoma Matropolitana.

Nájera, M. (coord.) (2013). Movimientos sociales, autonomía y resistencia. Guadalajara, México: Universidad de Guadalajara.

Rosales, G. (2015). Autonomía indígena en Bolivia: mecanismo de articulación y dispositivo de complejidad social. Sociológica, 30 (84): 143179. 
Schmidt-Hebbel, K. (2006). El Crecimiento Económico en Chile. Documento de Trabajo. Santiago, Chile:Banco Central de Chile.

Shapiro, R., \& Mahajan, H. (1986). Gender Differences in Policy Preferences: A Summary of Trends from the 1960s to the 1980s. Public Opinion Quarterly, 50, 42-61.

Sirlopú, D., Melipillán, R., Sánchez, A., \& Valdés, C. (2015). ¿Malos para aceptar la diversidad? Predictores socio-demográficos y psicológicos de las actitudes hacia el multiculturalismo en Chile. Psykhe, 42(1), 113.

Steeh, C., \& Schuman, H. (September de 1992). Young White Adults: Did Racial Attitudes Change in the 1980s? American Journal of Sociology, 98(2), 340-367.

Toledo, V. (2006). Pueblo Mapuche. Derechos Colectivos y Territorio. Desafio para la sustentabilidad democrática. Santiago, Chile: LOM.

Van Den Eynde, A., \& Dharmalingam, A. (2008). Public Opinion about Indigenous Australian Land Rights. New Zeland Sociology, 23(2), 90102.

Ward, C., \& Masgoret, A.-M. (2008). Attitudes toward Inmigrants, Inmigration and Multiculturalism in New Zeland: A Social Psychological Analysis. The International Migration Review, 42(1), 227-248.

Weakliem, D. (2002). The Effects of Education on Political Opinions: An International Study. International Journal of Public Opinion Research, 14(2), 141-157.

Recibido: 04.01.18

Aceptado: 12.04 .18 